\title{
Development of Acute Vogt-Koyanagi-Harada- like Syndrome during the Treatment Course with Vemurafenib for Metastatic Melanoma
}

\section{Atitaya Apivatthakakul, Paradee Kunavisarut, Aniki Rothova \& Kessara Pathanapitoon}

To cite this article: Atitaya Apivatthakakul, Paradee Kunavisarut, Aniki Rothova \& Kessara Pathanapitoon (2019): Development of Acute Vogt-Koyanagi-Harada-like Syndrome during the Treatment Course with Vemurafenib for Metastatic Melanoma, Ocular Immunology and Inflammation, DOI: 10.1080/09273948.2019.1597896

To link to this article: https://doi.org/10.1080/09273948.2019.1597896

曲 Published online: 04 Jun 2019.

Submit your article to this journal $₫$

Џ Article views: 37

View Crossmark data ¿ 


\title{
Development of Acute Vogt-Koyanagi-Harada-like Syndrome during the Treatment Course with Vemurafenib for Metastatic Melanoma
}

\author{
Atitaya Apivatthakakul, $\mathrm{MD}^{1}$, Paradee Kunavisarut, $\mathrm{MD}^{1}$, Aniki Rothova, $\mathrm{MD}, \mathrm{PhD}^{2}$, and Kessara \\ Pathanapitoon, $\mathrm{MD}, \mathrm{PhD} \mathbb{1}^{1}$ \\ ${ }^{1}$ Department of Ophthalmology, Faculty of Medicine, Chiang Mai University, Chiang Mai, Thailand and \\ ${ }^{2}$ Department of Ophthalmology, Erasmus Medical Centre, Rotterdam, The Netherlands
}

\begin{abstract}
Purpose: To report on ocular Vogt-Koyanagi-Harada (VKH)-like syndrome under vemurafenib treatment for metastatic melanoma.

Design: A case report.

Method: Description of clinical and imaging manifestations including fundus photography, fluorescein, and indocyanine green angiography.

Results: A 46-year-old Thai female was diagnosed with metastatic melanoma of the skin and had been treated with multiple surgical excisions, radiotherapy, and vemurafenib (initial dose $480 \mathrm{mg}$ orally twice daily, subsequently increased to maximum dose of $960 \mathrm{mg}$ twice daily). After 6 months of vemurafenib use, she complained of bilateral redness and photophobia and was diagnosed with bilateral anterior uveitis, which was topically treated. Two weeks later, her visual acuity (VA) sharply deteriorated to 20/80 and counting fingers. Ocular examination at that stage stronly resembled acute VKH disease. She exhibited intraocular inflammation, and her fundus examination revealed bilateral optic disc swelling and serous retinal detachment. Fluorescein angiogram showed disc leakage and multiple pinpoint hyperfluorescence leakage spots and indocyanine green demonstrated multiple hypofluorescent spots. Oral prednisolone $30 \mathrm{mg}$ /day was commenced while vemurafenib medication was ceased. Three weeks later, her vision improved, and serous retinal detachment subsided. However, her cutaneous melanoma recurred.

Conclusions: Vemurafenib, a potential adjunct treatment for metastatic melanoma, was complicated by the development of panuveitis, papillitis, and multiple serous detachments. These ocular symptoms were similar to the presentation of acute VKH syndrome.
\end{abstract}

Keywords: Exudative retinal detachment, melanoma, vemurafenib, Vogt-Koyanagi-Harada

Approximately half of cutaneous melanomas have a mutation in the BRAF gene, with V600E being the most common mutation, resulting in unchecked cell proliferation and increase of melanoma cell survival. In 2011, treatment of metastatic melanoma changed with the introduction of the targeted cancer therapy "BRAF inhibitors." Several studies have shown reduction in mortality rate and tumor progression with BRAF inhibitors. ${ }^{1}$ Vemurafenib, a selective and potent inhibitor of BRAF, was the first drug, which demonstrated the improvement of overall and progression-free survival.

The vast majority of BRAF mutations cause the activation of the mitogenic protein kinase (MEK), while vemurafenib interrupts a step on BRAF/MEK pathway and also affects immune system by modifying various cytokine production. Previously reported systemic side effects of vemurafenib include rash, arthralgia, photosensitivity, fatigue, nausea, diarrhea, squamous cell carcinoma, and keratoacanthoma of the

Received 2 February 2019; revised 18 March 2019; accepted 18 March 2019

Correspondence: Kessara Pathanapitoon MD, PhD., Department of Ophthalmology, Faculty of Medicine, Chiang Mai University, 110 Intawaroros Road, Chiang Mai 50200, Thailand. E-mail: kpathanapitoon@yahoo.com, kessara.pathana@cmu.ac.th

Color versions of one or more of the figures in the article can be found online at www.tandfonline.com/ioii. 
skin. ${ }^{1}$ In this case report, we report on a patient with metastatic cutaneous melanoma who developed signs of acute ocular Vogt-Koyanagi-Harada (VKH)-like syndrome during the treatment course of vemurafenib administration.

\section{CASE REPORT}

A 46-year-old Thai female was diagnosed with cutaneous melanoma at her right toe in February 2017 and has undergone several surgical excisions together with postoperative radiotherapy; however, these regimens could not control the melanoma progression. The melanoma continuously spread to her right thigh and groin. Since she has been BRAF mutation positive, she received the novel treatment modality with vemurafenib (initial dose $480 \mathrm{mg}$ orally twice daily and increased to maximum dose of $960 \mathrm{mg}$ orally twice daily for 6 months). After 6 months of vemurafenib treatment, she presented with acute anterior uveitis of her both eyes and was treated topically. Fundus examinations at that time were normal. After two weeks, she reported back with deteriorated visual acuity (VA) of 20/80 in $\mathrm{OD}$ and counting fingers in OS. She also complained of headaches, nausea, and tinnitus. Eye examination showed $3+$ cells in anterior chamber and $2+$ vitreous cells. Fundus examination revealed bilateral serous retinal detachment with choroidal folds and optic disc edema (Figure 1A, B). Optical coherence tomography showed subretinal fluid, which correlated with exudative detachment, choroidal thickening, and peripapillary subretinal fluid accumulation (Figure 1E, F). Fundus fluorescein angiogram showed multiple hyperfluorescent pinpoint spots with leakage together with disc leakage and subretinal pooling of fluorescein in the area of exudative retinal detachment (Figure 1C, D). Indocyanine green angiogram showed vascular leakage of choroid with late-phase multiple hypofluorescent dots. Ultrasonography showed choroidal thickening. The results of laboratory investigations, which included complete blood counts, venereal disease research laboratory test, treponema pallidum hemagglutination, human immunodeficiency virus antibody, tuberculin skin test, and chest X-ray, were all within the normal limits or negative. She was initially treated with oral prednisolone $60 \mathrm{mg} /$ day $(1 \mathrm{mg} / \mathrm{kg} /$ day $)$, but due to multiple side effects, the dose was quickly tapered down to $30 \mathrm{mg}$ /day together with posterior subtenon triamcinolone injection in both eyes while vemurafenib was withdrawn. Oral prednisolone was gradually tapered to $20 \mathrm{mg} /$ day. Her vision was improved in 4 weeks. Ten weeks after vemurafenib was withdrawn, the inflammation has subsided, and visual acuities improved to 20/30 OD and 20/40 OS. Unfortunately, her cutaneous melanoma recurred, and she had undergone further surgical excisions. Eight weeks after cessation of vemurafenib, she was diagnosed with brain metastasis.

\section{DISCUSSION}

We report on a patient with metastatic cutaneous melanoma, who developed panuveitis compatible with the diagnosis of acute VKH-like syndrome under vemurafenib therapy. Previously reported ocular complications related to vemurafenib medication included anterior uveitis, uveitic macular edema, and panuveitis; however, most of these reports were restricted to the anterior segment inflammation and responded well to topical corticosteroids. ${ }^{2}$ Our patient, however, demonstrated features that were similar to the acute phase of VKH disease.

VKH disease is a multisystem inflammatory disease characterized by bilateral panuveitis with serous retinal detachment. It is ascribed to an autoimmune inflammatory reaction mediated by $\mathrm{T}$-cells that target melanocyte in individuals susceptible to the disease and is often associated with skin, central nervous system, and auditory abnormalities. VKH-like development in melanoma patients was noted earlier, occurring after immunotherapy tumor-infiltrating lymphocytes and even after a surgical excision of metastatic melanoma. ${ }^{1}$ Donaldson et al. noted the presence of uveitis and vitiligo after bacillus Calmette-Guerin therapy for melanoma. ${ }^{3}$ The frequent occurrence of serous retinopathy after the use of vemurafenib and other BRAF inhibitors was repeatedly reported, however typically without any signs of intraocular inflammation. ${ }^{4}$ Other newer anti-melanoma drugs including dabrafenib and trametinib were also associated with the development of the VKH-like syndrome, manifesting ocular, skin, and hearing manifestations. Whether to discontinue treatment in patients with drug-induced uveitis is controversial and remains an individual decision. Based on Cancer therapy Common Terminology Criteria for Adverse Events (CTCAE), our patient was classified as a grade 4 ocular adverse event (panuveitis with VA of less than 20/200) and vemurafenib was withdrawn. In contrast, continuation of BRAF inhibitors or a dosage adjustment can be performed in patients with mild ocular side effects and a beneficial reaction to topical medication.

The exact cause of VKH-like ocular signs during BRAF therapy is not yet entirely explained, and several hypotheses have been postulated. First, the ocular inflammation may be caused by systemic oversensitization to melanocytic antigen during the treatment period as vemurafenib has a strong antitumor effect leading to massive apoptosis and death of melanocytes. Another hypothetical explanation might involve shared pathogenetic pathways common to skin melanoma and VKH disease, such as specific HLA types. Fujimura et al. reported the association between melanoma patients and 


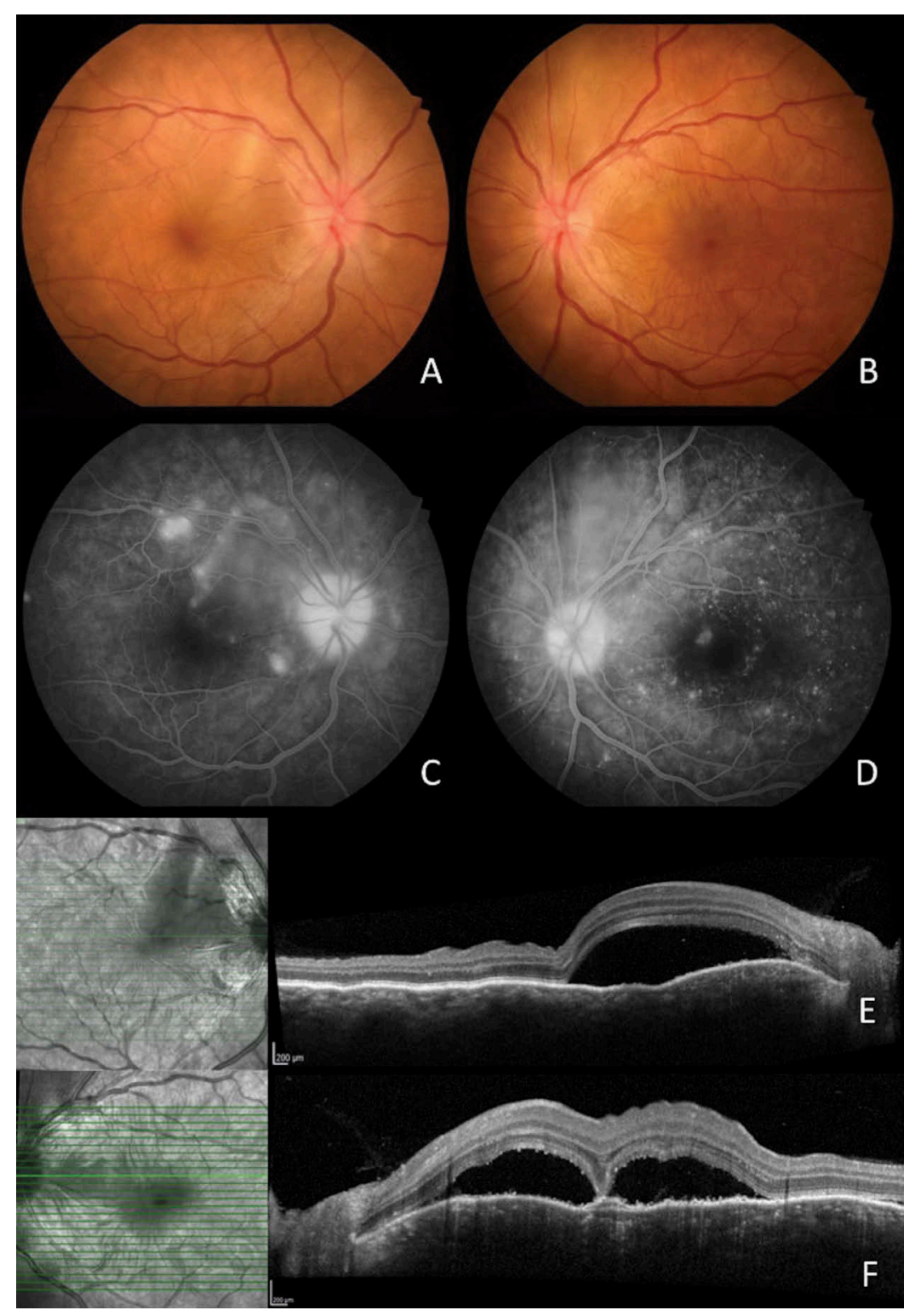

FIGURE 1. A, B: Bilateral serous retinal detachment, optic disc edema, and choroidal folds 6 months after start of vemurafenib treatment. C, D: Fluorescein angiography shows disc leakage and multiple hyperfluorescence leakage pinpoint lesions with associated and subretinal pooling of fluorescein in the area of exudative detachment compatible with acute Vogt-Koyanagi-Harada disease. E, F: Optical coherence tomography shows bilateral serous subretinal fluid accumulation with subretinal septa, subretinal hyperreflective dots, and retinal folds visible in the left eye.

HLA-DRB1*04:05, which is also strongly associated with VKH disease. ${ }^{5}$ In summary, vemurafenib is one of the life-prolonging medications for patients with metastatic melanoma but might be associated with ocular side effects ranging from mild to severe inflammation. The discontinuation of this valuable drug should be based on the severity of adverse effects. Further studies are needed to evaluate the potential treatment options for severe ocular adverse events.

\section{CONFLICT OF INTEREST}

All authors report no conflict of interest.

\section{ORCID}

Kessara Pathanapitoon (1) http://orcid.org/00000003-4447-1704

\section{REFERENCES}

1. Chapman PB, Hauschild A, Robert C, et al. Improved survival with vemurafenib in melanoma with BRAF V600E mutation. N Engl J Med. 2011;364(26):2507-2516. doi:10.1056/NEJMoa1103782.

2. Guedj M, Queant A, Funck-Brentano E, et al. Uveitis in patients with late-stage cutaneous melanoma treated with 


\section{A. Apivatthakakul et al.}

vemurafenib. JAMA Ophthalmol. 2014;132(12):1421-1425. doi:10.1001/jamaophthalmol.2014.3024.

3. Donaldson RC, Canaan SA Jr., McLean RB, Ackerman LV. Uveitis and vitiligo associated with BCG treatment for malignant melanoma. Surgery. 1974;76:771-778.

4. de la Cruz-Merino L, Di Guardo L, Grob JJ, et al. Clinical features of serous retinopathy observed with cobimetinib in patients with BRAF-mutated melanoma treated in the randomized coBRIM study. J Transl Med. 2017;15(1):146. doi:10.1186/s12967-017-1246-0.

5. Fujimura T, Kambayashi Y, Tanita K, et al. HLA-DRB $1 * 04$ :05 in two cases of Vogt-Koyanagi-Harada diseaselike uveitis developing from an advanced melanoma patient treated by sequential administration of nivolumab and dabrafenib/trametinib therapy. J Dermatol. 2018;45(6):735-737. doi:10.1111/1346-8138.14273. 